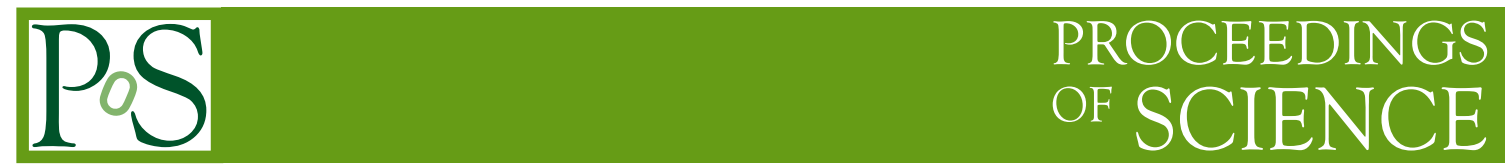

\title{
ATLAS Virtual Visits - Take part from anywhere in the world
}

\section{Muhammad Alhroob ${ }^{1}$}

University of Oklahoma,

Norman, Oklahoma, USA

E-mail: muhammad.alhroob@cern.ch

This proceeding presents a summary of the ATLAS Virtual Visit service: it discusses the booking system, the video conference from the ATLAS Visitors Centre, the opportunity to make virtual tours from the ATLAS cavern, and the new system that the ATLAS collaboration will install in the ATLAS cavern to enhance the underground Virtual Visits.

The Eighth Annual Conference on Large Hadron Collider Physics-LHCP2020 25-30 May, 2020

online

${ }^{1}$ On behalf of the ATLAS Collaboration. 


\section{Introduction}

This year, the ATLAS collaboration at the LHC celebrates the tenth anniversary of the Virtual Visits programme. The collaboration uses this popular and effective method to bring the excitement of scientific exploration into classrooms and public places around the world. Tens of thousands have already taken part in the programme, with many languages, from tens of countries covering the six populated continents.

This programme uses a combination of video conferencing, webcasts, and video recording to communicate with remote audiences. A Virtual Visit typically lasts about an hour and consists of two separate parts:

- Guides briefly introduce themselves, how they came to work in particle physics at the ATLAS experiment [1] at the LHC. They talk about the LHC and ATLAS detector, and also they highlight the physics results and give examples of searches taking place in ATLAS.

- A longer discussion between the guides and the remote audience on various aspects linked to the ATLAS experiment.

By the end of the tour, visitors have an idea about CERN, the LHC, the ATLAS experiment and the goal of the collaboration in exploring the building blocks and fundamental forces of the universe.

\section{Technical information}

Interested groups can request Virtual Visits matching their needs and preferences using a simple booking form [2] that they can send to the virtual visit team to reserve time slots and to explain their expectations. The Virtual Visit coordinator finds guides that best suit the request. Occasionally, the coordinator may invite additional guides or experiments to join the video conference. Also, the coordinator looks for a trained operator from the collaboration to provide the technical support, e.g. create a virtual room, help to test the hardware in the remote site, support the guides in running the cameras, broadcasting and recording the visit for later publication.

Guides use video-conferencing applications that participants can download freely. They primarily use VidyoConnect [3], but can also use ZOOM [4] or Skype [5]. The primary location where the guides stand is the ATLAS Visitors Centre next to the ATLAS Control Room. There are two pre-installed HD cameras:

- One camera in the Visitors Centre focuses on the guides.

- The other camera provides panoramic views from inside the ATLAS Control Room.

Guides also can utilise their laptops, tablets and smartphones to share media content. The ATLAS Collaboration is upgrading the Visitors Centre and installing a new system in the ATLAS cavern to allow guides to perform visits more frequently and with higher quality next to the ATLAS detector. Figure 1 represents a photo that was captured during a virtual tour from the ATLAS Visitors Centre in 2014 with Al-Mansoura University, Egypt. 


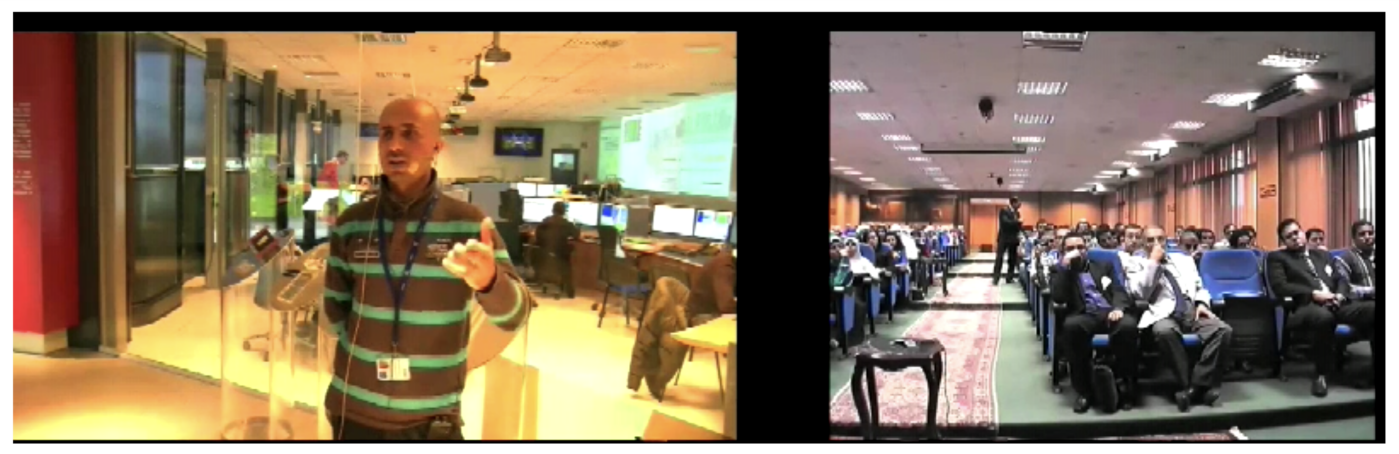

Figure 1: Al-Mansoura University, Mansoura, Egypt, shown participating in an ATLAS guided tour from the ATLAS Visitors Centre, with Muhammad Alhroob acting as a guide, October 27, 2014 [6].
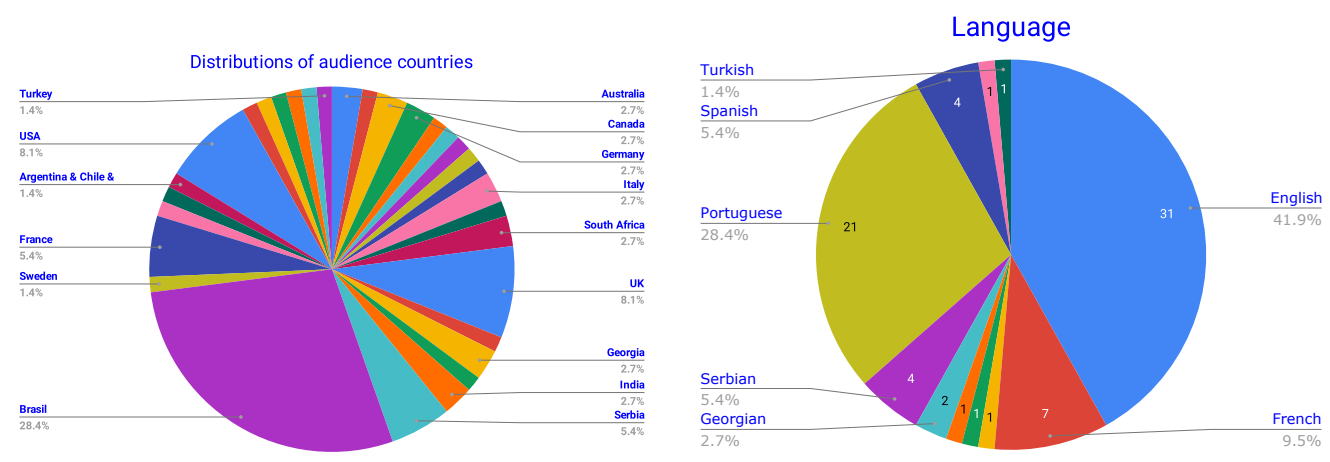

Figure 2: Right, the participated countries in the ATLAS Virtual Visits in 2019. Left, the various languages used by the guides.

\section{The reach of the Virtual Visit programme}

Any group can request a virtual visit:

- Schools and universities,

- Pupils, undergraduate and graduate students,

- Science and non-science classes,

- The ATLAS Collaboration also offers virtual tours to public events and science festivals.

In 2019, the collaboration offered over 75 visits, of which guides streamed many from the ATLAS cavern. Participants were from over 31 countries across the six populated continents, with a wide range of languages such as English, Portuguese, French, Spanish, Serbian, Georgian, Arabic and others. Figure 2 left, shows the distribution of participating countries in the ATLAS Virtual Visits in 2019, while Figure 2 right, shows the various languages used in those visits. Guides regularly livestream visits to allow people unable to connect to the primary room to follow it. Also, if possible, guides record the visit to publish it later on the CERN Document Server to allow more people to benefit from the activity. 


\section{References}

[1] ATLAS Collaboration, The ATLAS Experiment at the CERN Large Hadron Collider, JINST3 (2008) S08003

[2] The ATLAS Virtual Visits portal, https://atlasvirtualvisit.web.cern.ch/ content/prepare-your-visit

[3] VidyoConnect IP-based video conferencing software, https://www .vidyo.com

[4] ZOOM IP-based video conferencing software, https://www . zoom.us/

[5] Skype IP-based video conferencing software, https: //www. skype.com/

[6] ATLAS Virtual Visit with Mansoura University, https://atlasvirtualvisit.web. cern.ch/content/mansoura-university

[7] The CERN Document Server, https://cds.cern.ch 